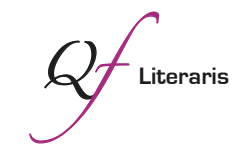

\title{
Il catalogo muliebre nella Napoli del XVI secolo: un contributo di Benedetto Croce
}

\author{
The catalogue of women in sixteenth-century Naples: \\ A contribution by Benedetto Croce
}

\section{Roberta Angelica Ruotolo}

Università degli studi di Napoli Federico II. ro.ruotolo@gmail.com Recibido: 26/06/2017. Aceptado: 09/09/2017

Riassunto: La letteratura napoletana è ricca testi incentrati sulle lodi delle donne della città, genere che si vitalizza a contatto con il vivace quadro della società aristocratica del Vicereame. È Benedetto Croce, coadiuvato da Giuseppe Ceci, ad occuparsi tra i primi di quella serie di rime, poemetti, capitoli ed epigrammi incentrati sul catalogo muliebre perché consapevole del loro apporto alla storia del costume e della letteratura nella Napoli del XVI secolo. In Lodi di dame napoletane del secolo decimosesto vengono analizzate quelle opere che la nobiltà napoletana e spagnola si beava di leggere vedendo in queste, e nella raffinata lingua petrarchesca in cui erano scritte, uno specchio della propria elegante condotta.

Parole chiave: catalogo muliebre; letteratura napoletana; XVI secolo.

\begin{abstract}
In Neapolitan literature there are many literary works whose focus are the praises of women of Naples, this genre vitalizes in contact with the aristocratic society of the Viceroyalty. Benedetto Croce, assisted by Giuseppe Ceci, has been involved in the early series of rhymes, poems, chapters and epigrams focused on the women's catalog, aware of their contribution to the history of costume and literature in Naples in the 16th century. In Lodi di dame napoletane del secolo decimosesto are analyzed those works that the Neapolitan and Spanish nobility would like to read because they saw in them, and in the refined language in Petrarchan style, in which they were written, a mirror of their elegant behavior.
\end{abstract}

Keywords: women's catalogue; neapolitan literature; XVI century. 



\section{Introduzione}

Nel 1894 veniva pubblicato a Napoli un prezioso opuscolo intitolato Lodi di dame napoletane del secolo decimosesto dall' "Amor prigioniero" di Mario Di Leo, con notizie ed estratti di altri poemetti sincroni di simile argomento a cura di Benedetto Croce e Giuseppe Ceci. L'opera presenta come introduzione ${ }^{1}$ una dettagliata ricognizione dell'evolversi del genere di produzione del catalogo muliebre entro l'ambito della letteratura napoletana cinque-seicentesca che ha il merito di offrire al lettore sia notizie su opere ed autori prima sconosciuti, o quasi, che considerazioni sulla società di quei tempi. Seguono alcune delle ottave tratte dal "leggiadro" poemetto L'Amor prigioniero (1538) di Mario Di Leo, opera che a parere del Croce può vantare pregi per forma ed invenzione, ed infine le note biografiche inerenti alle dame menzionate proposte da Giuseppe Ceci.

Dal Tempio d'Amore (1536) di Iacopo Campanile (detto Capanio) allo Specchio de le bellissime donne napoletane (1536) di Iacomo Beldando e il Triompho di Carlo Quinto (1536) di Giovan Battista Pino, per citarne solo alcune, si tratta di opere composte o stampate in occasione della venuta dell'Imperatore Carlo V a Napoli con l'obiettivo della nobiltà napoletana di celebrare se stessa attraverso i propri condottieri e nobili o la mediazione delle loro dame.

La visita dell'Imperatore segnò una fase cruciale per quanto riguarda la storia politica e letteraria del Regno: da un lato, baroni, patriziato cittadino e borghesia napoletana, dopo l'entusiastica adesione all'impresa di Tunisi, cercarono vanamente di liberarsi dell'odiato viceré di Toledo (D'Agostino, 1967; Galasso, 2006); dall'altro, la presenza dell'Imperatore stimolò gli omaggi dei letterati del tempo risollevando le sorti dell'editoria ${ }^{2}$ che fece del progetto propagandistico della nobiltà napoletana uno dei suoi principali interessi, segnando di fatto l'acme della cultura umanistico-rinascimentale napoletana ${ }^{3}$. La città visse durante l'inverno che Gregorio Rosso disse "una continua primavera" un

\footnotetext{
${ }^{1}$ L'introduzione è stata, con qualche modifica, ripubblicata in Croce, [1942] 1953: 31929.

${ }^{2}$ Cfr. Sull'editoria napoletana nel XVI secolo: Ascarelli, 1953; Ascarelli- Menato, 1989; Bresciano, 1936; Giustiniani, 1793; Santoro, 1997; Toscano, 1992; Zappella, 1984.

${ }^{3}$ Per un quadro sulla letteratura napoletana nel Cinquecento: De Blasi \& Varvaro, 1988; Dionisotti, 1963; Petrocchi, 1967.
} 
susseguirsi di occasioni mondane, feste, banchetti, ricevimenti, giostre e tornei e tra queste venne celebrato anche il matrimonio della figlia naturale di Carlo V, Margherita d'Austria con Alessandro de' Medici duca di Firenze: Lo specchio de le bellissime donne napoletane dedicato a Margherita già duchessa di Firenze, ne è appunto un omaggio.

Pur entro la forma di stereotipate lodi, i poemetti napoletani offrono un campione della società del tempo fornendo informazioni biografiche di valore storico e, allo stesso tempo, riguardo alla forma, dati sulla ricezione in area napoletana del metro dell'ottava e della codificazione linguistica proposta dal Bembo.

Proprio il metro, l'ottava, è quello distintivo della narrativa epica, mitologica ed encomiastica, in questo caso piegato alle esigenze del topos catalogico di uomini e donne illustri attraverso il travestimento o l'allegoria mitologica, l'allegoria del tempio-palazzo d'Amore o del viaggio ultraterreno. Come la terzina incatenata, l'ottava rima è un metro narrativo ed è attraverso poemetti come il Filostrato, il Teseida, il Ninfale fiesolano, che si diffonde quella "letteratura divertente ricca di amori e avventure, di ampia diffusione presso il pubblico non specialistico di media cultura" (Bruni, 2002: 123) e soprattutto femminile. Le donne sono le destinatarie reali ed ideali, pensiamo alla posizione incipitaria del termine nel primo verso del Furioso, che traggono piacere dalla lettura di opere-specchio o di invenzione, "per motivi quasi pornografici" (Bruni, 2002: 123).

Nella letteratura napoletana abbondano testi incentrati sulle lodi delle donne della città, genere questo di chiara derivazione toscana dalla pistola dantesca alla Caccia del Boccaccio, che "si vitalizza a contatto col ricco vivace quadro della società aristocratica del Vicereame" (Petrocchi, 1967: 305). Grazie al contributo di Giuseppe Ceci e Benedetto Croce la serie di rime, poemetti, capitoli ed epigrammi, tutti incentrati sul catalogo muliebre, dalle prime composizioni della Napoli aragonese di Suero de Ribera e Juan de Tapia ai seicenteschi anagrammi di Basile, hanno trovato un'adeguata analisi. Si tratta di testi composti per "galanteria cavalleresca e adulazione cortigiana" (Ceci \& Croce, 1894: IX) che la nobiltà napoletana e spagnola si beava di leggere vedendo in questi, e nella raffinata lingua petrarchesca in cui erano scritti, uno specchio della propria elegante condotta. Non il "culto cristiano-germanico della donna [...] quello che rappresenta una strana complicazione, $\mathrm{o}$ contaminazione, dell'istinto sessuale con le più pure idealità morali 
dell'uomo" (Ceci \& Croce: 1894: X) ma vuota e rituale galanteria, una ripetitiva recita come formulari e ripetitivi erano questi componimenti dove i ritratti delle donne sembrano rifrangersi gli uni negli altri. Quanto alla forma, Petrarca fu modello indiscusso del XVI secolo "perché l'epoca galante, raffinata e cortigiana credette di aver trovato in questo poeta dell'amore il rappresentante ed il campione dei suoi sentimenti... dei suoi ideali [...]" (Borzelli, 1888: 75).

\section{Prime prove del catalogo muliebre}

\subsection{Le origini classiche}

Il genere del catalogo (dal gr. katálogos 'elenco') affonda le radici nel mondo classico. Nel secondo libro dell'Iliade, Omero per dare il senso dell'immensità dell'esercito greco che sbarca ad assediare Troia, nomina i capitani e le navi dello schieramento ed ecco quindi il celebre $\mathrm{Ca}$ talogo delle navi (II, vv. 494-759), il capostipite di questo genere che si arricchisce con quello delle donne nell'undicesimo canto dell'Odissea e quello delle eroine attribuito ad Esiodo (Catalogo delle donne, I-V), in cui si identificano le Eee o Eoie, elenco di miti riferiti al nome delle

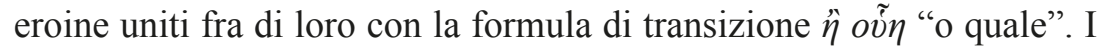
cataloghi risultano essere luoghi poetici dalla struttura altamente formalizzata e tradizionale ma allo stesso tempo flessibile perché ampliabile all'infinito.

\subsection{Il modello boccaccesco}

La struttura rimane invariata ma cambia l'epoca: è il Trecento e si tratta della prima prova letteraria del Boccaccio, la Caccia di Diana, un poemetto composto intorno al 1333-34, che consta di 18 canti in terzine incatenate. È primavera e il poeta innamorato cercando conforto alle sue pene si rifugia in una valle dove un gruppo di nobildonne napoletane sono impegnate in una battuta venatoria in onore di Diana. Al momento di offrire le prede a Giove, secondo la volontà della dea, le nobildonne si rifiutano di eseguire il sacrificio, invocando l'autorità di Venere. Si assiste allora ad una metamorfosi degli animali catturati in giovani-amanti, decretando la vittoria finale della dea dell'amore. 
Dietro la costruzione narrativa e allegorica c'è l'obiettivo, tutto galante, di celebrare le nobildonne partenopee e proiettare la raffinata vita della corte angioina in una dimensione letteraria e sovratemporale. Il catalogo delle donne napoletane recupera il precedente modello dantesco della pistola in forma di sirventese scritta per celebrare le sessanta più belle donne di Firenze (Vita Nova, VI) ma bisogna ricordare anche che catalogo muliebre vantava un'illustre tradizione medievale, quella dei sirventesi e delle "visioni d'amore"4.

\subsection{La lode degli spagnoli}

Sempre scritta a Napoli, ma da autori stranieri, è la serie di componimenti della raccolta detta Cancionero de Estúñiga (XV sec.). È la Napoli di Alfonso d'Aragona, il "principe letterato" che amò circondarsi di intellettuali italiani e spagnoli sia per proprio culto delle lettere che per un progetto di politica culturale (De Blasi \& Varvaro, 1988). Presentano lodi di donne napoletane le poesie di Suero de Ribera e Juan de Tapia. Nel caso del primo, il componimento ${ }^{5}$ dedicato a Francesco Centelles conte di Oliva, è aperto da una cobla introduttiva dove il poeta dice di aver visto donne più belle delle napoletane, tranne le sei che andrà poi ad elencare: si tratta della contessa di Aderno, una donna della famiglia Gattula, Lucrezia d'Alagno del seggio di Nido, una Camilla del seggio Capuana, una Lucrezia e Margherita Minutolo. Si veda ad esempio questa cobla:

Sin más quedar en olvido valor que tanto se precia es la fermosa Lucrecia del gentil sege de Nido: donzella de grand valia,

\footnotetext{
4 "(la tradizione) dei sirventesi e delle visioni d'amore, che galantemente presentano schiere di dame gentili[...] risale alla più autorevole letteratura d'amore d'oltralpe: alle insistenti figurazioni di schiere di donne nel De Amore di Andrea Cappellano, nel Roman de la Rose e in derivati diretti come Le Fablel dou Dieu d'Amors e De Venus la deesse d'amor; al 'genere' dei francesi 'tournoiments des dames' e ai componimenti affini provenzali (come il Carros di Rambaldo di Vaquieras, La Treva di Guilhem de la Tor e la Mesclansa et batalha di Aimeric de Peguilhan) e anche ai così detti 'giardini d'amore' o alle stesse 'corti d'amore' e così via" ( Branca, 1967: 6-7).

${ }^{5}$ Cito dall'edizione curata da Periñán, 1968.
} 
en estremo singular,

por quien dizen el cantar:

"Para mi me la querría" (Poesías, XIV, vv. 41-49).

Il poeta con la sua "flaca memoria" cerca di rendere onore alla bellezza di queste donne che, come prevede il genere, è sia fisica che morale:

donzella bien paresciente

en superlativo grado,

compassada su belleza;

segund el mi parescer

bien creo que deve ser

thesoro de gentileza (Poesías, XIV, vv. 59-64).

Quello di Juan de Tapia è un altro dezir ${ }^{6}$ dove vengono celebrate le donne di Tropea ("lodando e nombrando todas las damas de Turpia") e del Regno. Composto durante il soggiorno del poeta in questa città (1445-49), nelle prime coplas viene descritta la sua condizione di esule in terra straniera, segue l'enumeratio delle donne ("Quise las damas loar / Perdiendo la mi desfierra" (vv. 7-8). I riferimenti sono molto generici da Volcana a la Toralda, da Castana a D'Aflito, le dame vengono menzionate per il loro cognome e descritte con brevissimi cenni mediante paragoni a figure del mito o facendo un breve riferimento alle loro vicende biografiche. Canonico il lessico delle lodi:

Avlatas son las estrellas, e la Sava es la luna, Antonela Pata es una muy fermosa entre ellas. El sol que alumbra las bellas es mi dama por amores. e mejor de las meiores a mi parescer doncellas (Poemas, vv. 33-40).

\footnotetext{
${ }^{6}$ Cito dall'edizione curata da Giuliani, 2004.
} 


\subsection{Il catalogo e l'ottava: l'Orlando Furioso a Napoli}

Nel caso dell' Orlando Furioso si tratta di un'opera composta fuori dal Regno $^{7}$ ma, come vedremo, in stretta relazione con la corte napoletana. La galleria di ritratti femminili del canto XLVI (ott. 3-10) e le lodi a Isabella (ott. 9-11) e Vittoria Colonna (ott. 16-20) del canto XXXVII sanciscono di fatto il connubio tra l'ottava e il catalogo muliebre che avrà molta fortuna a Napoli dove il Furioso circolava entro la corte degli Ávalos (Toscano, 2000) e la cui lingua viene proposta come modello, accanto a quella delle "tre corone", da Benedetto di Falco (1535) e Fabricio Luna (1536) per una canone aperto ad autori della letteratura coeva $^{8}$, mentre nel frattempo Dianora Sanseverino scriveva Stanze sopra una stanza di messer Ludovico Ariosto [...] (1545) e Laura Terracina il Discorso sopra tutti li primi canti d'Orlando Furioso (1549 colophon 1550).

\section{Le bellissime donne napoletane: il genere del laus mulieris a Napoli (1536-1605)}

Tra i primi poemetti-catalogo ad esser stati scritti a Napoli con finalità encomiastiche, c'è quello di Iacopo Campanile: il Tempio d'Amore'. Proprio Croce dà per primo notizia del pometto e, sulla scia delle ipote-

\footnotetext{
${ }^{7}$ Altra opera cinquecentesca in ottave ascrivibile al genere del laus mulieris è di Claudio Tolomei, Laude delle donne Bolognese (Bologna, Rubera, 1514). Si tratta di un catalogo di bellezze felsinee strutturato in 281 ottave suddivise in tre libri: nel primo viene descritto un giardino edificato dalle Grazie; il secondo è interamente dedicato alla celebrazione di 21 donne bolognesi; il terzo racconta invece la storia di Amore che, inviato dalla madre Venere a far innamorare le custodi del giardino, diviene prigioniero delle donne.

${ }^{8}$ Il Di Falco nel suo Rimario propone un canone di dieci autori (Petrarca, Boccaccio, Dante, Ariosto, Pulci, Sannazaro, Bembo, Landino, Machiavelli, Castiglione), mentre il Luna nel suo Vocabulario di cinquemila vocabuli toschi non meno oscuri che utili e necessari propone l'Ariosto del Furioso, Boccaccio, Petrarca, Dante come recita il titolo ed altri autori utilizzati per il risconto delle singole voci come l'Aretino e il Sannazaro. ${ }^{9}$ L'opera è conservata manoscritta in un miscellaneo segnato XIII. G. 42. della Biblioteca Nazionale di Napoli intitolato Raccolta di Poesie diverse (cc. 231r-241v), qui le ottave hanno un ordine diverso rispetto alla stampa alifana (ott. 46-61, 10-45, 4-9). La princeps è intitolata Opera nuova nomata Vero tempio de Amore (Alife, Acilio, 11 giugno 1536). Un'edizione del poemetto è stata curata da Altamura, 1980. Cito dall'edizione di Altamura.
} 
si del Pèrcopo, colloca la sua produzione nel XVI secolo. L'opera venne scritta probabilmente negli anni Venti del Cinquecento e poi data alle stampe nel 1536 ad Alife; introdotta da tre epistole, una dell'editore alla contessa d'Alife, due dell'autore ad Altobello d'Ischia e alla duchessa di Francavilla, consta di 61 ottave. Per Altamura, che ne ha curato un'edizione critica:

Il poemetto - sia l'originale, sia la rielaborazione del Franco ${ }^{10}$ - è in sé assai povera cosa, tutto contesto com'è delle solite illimitate lodi sia alla bellezza che alla virtù delle donne che, assai spesso, non furono né belle né virtuose: il tutto retoricamente illeggiadrito da petulanti e stucchevoli reminiscenze mitologiche non sempre appropriate (Altamura, 1980: 8-9).

Il testo, dedicato a Costanza d'Ávalos, duchessa di Francavilla (che non figura tra le donne-colonne), è aperto da ottave (1-13) che forniscono il motivo che porta all'edificazione del Tempio: Cupido, trafitto da una freccia di Momo, prova invidia di questi e decide di rivolgersi a Giove per aver un tempio dove "locar gli alti trofei". Ottenuto il consenso del padre degli dèi sceglie Napoli "che gli diletta la memoria" e alle falde di Posillipo costruisce il suo tempio con trenta donne-colonne. La prima è Isabella di Requesens poi la marchesa di Pescara, Vittoria il cui cognome era di sostegno all'edificio allegorico creato dall'autore; le due fanno da capo-fila alle schiere di quindici donne che seguono.

Amore "elegge" o "ritolse" le donne, i cui unici riferimenti sono quelli canonici alla loro vista che fa innamorare o alla bellezza pari a quella di Venere che disorienta il faretrato dio. Non sembra esserci un preciso ordine o gerarchia tra le colonne ("le ho messe come la fiacca memoria me dittava") tutte poste sul medesimo piano di perfezione, si veda ad esempio:

Per seguir il suo degno e bel lavoro

Iulia Carrafa a llui se loca avante, colonna salda e non di gemme ed oro, ma di vera beltà novo sembiante; tal che chi vuol veder grazia e decoro

\footnotetext{
${ }^{10}$ Altamura analizza anche il plagio realizzato da Niccolò Franco con il medesimo titolo, opera stampata a Venezia, per Francesco Marcolini da Forlì nell'agosto 1536 (Altamura, 1890).
} 
miri costei dal bel capo a le piante e chiar vedrà quanto natura ha forza vedendo il ciel sotto terrena scorza (Tempio, ott. 26).

Segue poi il racconto dell'allestimento del tempio con fondamenta di speranza, tetto di onestà, altari, campane, sacerdoti, ecc. (ott. 45-61).

In Beldando e nei successivi autori, manca qualsiasi nota esplicativa a giustificazione della scelta del metro, che invece troviamo nel Tempio d'Amore dove il Capanio nella dedica ad Altobello d'Ischia ${ }^{11}$ si avvale dell'autorità del lombardo Carlo Agnello e di altri autori che in quella regione adoperavano l'ottava rima perché probabilmente negli anni Venti del Cinquecento, scrivere in ottave veniva avvertito come un "esotismo metrico settentrionale" (Parenti, 1995: 131).

Lo specchio de le bellissime donne napoletane (1536), Il Triompho di Carlo Quinto a cavallieri et alle donne napoletane (1536) e L'Amor prigioniero (1538) sono un gruppo di opere, tutte edite da Ioanne Sultzbach. Originario di Imola, Iacomo Beldando giunse a Napoli, presso il circolo di Vittoria Colonna nel 1533 grazie alla raccomandazione di Claudio Tolomei (Ferrero \& Müller, 1892: 86-87), probabilmente aspirando al ruolo di poeta di corte. Purtroppo le informazioni su quest'autore non sono molte dacché lo stesso Manzi (Manzi, 1970: 67) nei suoi Annali afferma che i biografi tacciono su questo "brillante poeta" e storici letterari come Mazzuchelli (Mazzuchelli, 1753: 623), e quelli che alla sua opera si rifanno, si limitano a menzionarlo come autore dello Specchio. In realtà il Beldando fu autore di un poemetto in ottave, Lo specchio de le bellissime donne napoletane e di un Rimario.

Lo Specchio ${ }^{12}$, un poemetto di 109 ottave introdotto dalla lettera di dedica a Margherita d'Austria, è incentrato sulla descrizione del viaggio dell'autore al cielo di Venere e su quella delle 79 nobildonne napo-

\footnotetext{
11 " [...] escuseraime ancora del stile de scriverlo in stanze [...] che a questo me ha spronato la auttorità del nostro candido Messer Carlo Agnello e de molti dottissimi scrittori che volentiero in Lombardia usano questo stile". Ms. XIII. G. 42 (Biblioteca Nazionale di Napoli), c. 231r.

${ }^{12}$ L'opera venne stampata in Napoli per Ioanne Sultzbach il 19febbraio 1536. Altamura nella terza serie di Curiosità letterarie napoletane (1972: 17-62) pubblica un'edizione interpretativa dello Specchio non esente da sviste e incisive alterazioni del testo al punto da modificarne la veste linguistica ed intaccarne la struttura metrica. De Nichilo, 2006: 351-380, ha ripreso e proposto un'analisi del poemetto. Cito da Ruotolo, in corso di pubblicazione.
} 
letane che incontra, identificate con l'aiuto della sua 'Beatrice', le cui anime attendono di scendere sulla terra per mostrare al mondo la grandezza di Amore. Costanti puntelli alla narrazione sono le dichiarazioni di inadeguatezza del poeta: la lingua umana non riuscirà mai "con simplici parole" ad esprimere la grandezza delle madonne che vede, la loro bellezza fisica unita alla perfezione morale. Curioso è il petrarchismo che traspare da queste ottave dove Caterina Sanseverino viene descritta come più bella della Laura di Petrarca:

Quinci non longe humanamente altera

vidi la bella donna saggia, tanto

che se col buon Toscan già venut'era,

togliea de la bellezza a Laura il vanto (Specchio, ott. 55, vv. 1-4).

Sappiamo che Croce era in possesso di una copia dello Specchio perché regalatagli da Dionisotti che "per caso aveva acquistato su una bancarella una rara cinquecentina, Lo specchio delle bellissime donne napoletane del Beldando, un testo che a tutti gli effetti nei circuiti della storia letteraria era considerato una scoperta di Croce" (Fera, 2001: 69) e benché fosse duro il giudizio del critico partenopeo per i suoi versi "assai più rozzi di quelli del Di Leo" (Croce, 1953: 325) quanto per il suo scarso valore storico, il poemetto del Beldando, come altri, offre una fonte di informazioni sulla Napoli cinquecentesca.

Napoli in quel periodo, infatti, si distinse per 'l'avanguardia' del protagonismo femminile della sua corte (Costanza d'Avalos, Vittoria Colonna, Giulia Gonzaga, Maria d'Aragona, Isabella Villamarino per menzionarne alcune): invano si cercherebbero in altre città donne di corte e di maneggi politici e di spiritualità profonda come quelle appena ricordate (Toscano, 2000).

A tal proposito Toscano in un suo contributo (2000) menziona un opuscolo, Cose volgare di messere Augostino Landulfo [...] nelle quale se raggiona dell'una e l'altra fortuna [...] (1536) ${ }^{13}$, descrizione di un banchetto organizzato dai nobili napoletani nel mese di dicembre per festeggiare le future nozze di Margherita dove si esibirono quattro gruppi di paggi e quattro di giovinette cantando componimenti poetici con accompagnamento musicale.

${ }^{13}$ L'opuscolo è molto raro l'unica copia a stampa censita è stata stampata a Napoli per M. Cancer. Cfr. Sull'opera e l'autore: Dilemmi, 2000; Manzi,1972; Toscano, 2000. 
La brigata, formata di uomini, [...] è integrata con ruolo non passivo da "altrettante di sagge donne, e delle prime di bellezza e di sangue di Napoli” (c. B2r-v), la cui presenza nel contesto della festa appare complessivamente paritaria rispetto a quella maschile (Toscano, 2000: 251).

Le donne quindi erano sia produttrici e ispiratici di letteratura che partecipi della vita politica del tempo. Rilevanti, infatti, sono le stanze dello Specchio che presentano le donne caratterizzandole per le loro competenze politiche reali ${ }^{14}$ o ideali ${ }^{15}$ o le loro doti poetiche ${ }^{16}$, che si siano abbeverate alla fonte dell'ispirazione poetica o abbiano la testa cinta di corone di alloro, si veda ad esempio:

Sen gíà l'alta regina in vest'oscura

coronata di lauro a passi lenti

dicendo: "Io son Collonna alma Vittoria

che canta ogni pregiata et dotta istoria" (Specchio, ott. 98, vv. 5-8).

Sia Il Triompho che L'Amor prigioniero sono ambientati in una Napoli più o meno stilizzata dal mito: nel primo ad elementi fantastici quali ninfe e sirene si aggiungono elementi di topografia reale come $\mathrm{i}$ seggi di Napoli; il secondo descrive con brevi pennellate degli scorci della città che tanto piacque a Giove e fa da sfondo alla lotta di Cupido e le guerriere napoletane. Nel Triompho ${ }^{17}$ il Pino inserisce il catalogo di nobildonne e cavalieri entro un racconto che ha come protagonista

\footnotetext{
${ }^{14}$ Si tratta di Antonia Del Balzo e Maria di Capua: "Quelle altre due che vedi andar insieme / ragionando d'amor, d'arme e di stato" (Specchio, ott. 43, vv. 1-2); Lucrezia e Geronima Frangina e Giovanna Spina: "Ecco tre donne honestamente altere, / di sangue, d'amor giunte et han dal cielo / quanto di buon, quanto di bello havere / si possa, ove ha poi forzza il caldo e il gielo / et con l'oro et col senno harian potere" (Specchio, ott. 87, vv. 1-5).

${ }^{15}$ Isabella Vigliamarina: "che Natura le diè l'alto governo, / non vedendo di lei cosa più bella" (Specchio, ott. 24, vv. 6-7); Isabella Colonna: “et può sì col valor, con 1'alto ingegno / che può por nove leggi a novo regno" (Specchio, ott. 32, vv. 7-8).

${ }^{16}$ Rappresentate con corone d'alloro Susanna Gonzaga (ott. 47) e Vittoria Colonna (ott. 98); Maria di Cardona su un carro "rugiadoso / di nectare e d'ambrosia" (ott. 45); Isabella Caracciolo che beve alla fonte di Pegaso (ott. 42); Maria Sanseverino che "orna d'Apollo il sacro speco" (ott. 57); Lucrezia Scaglione è amica delle Grazie (ott. 64).

${ }^{17}$ La princeps venne pubblicata da Sultzbach a Napoli 1'8 settembre 1536. L'opera consta di tre canti ed è in ottave. Cito dall'esemplare custodito nella Biblioteca universitaria di Napoli (RARI 0012 002), cc. 1-74.
} 
reale, l'autore ed ideale, Carlo V: l'Imperatore viene evocato costantemente come portatore di pace e nuovo Cesare e ciò impedisce ogni slancio originale del testo perché tutto è volto ad un fine propagandistico $^{18}$. Il poeta dapprima miracolosamente disceso nel letto del fiume guidato da una ninfa, visita il tempio di Sebeto, poi dietro le indicazioni di un" "angelica sirena" visita la città ed assiste ai festeggiamenti indetti per la venuta dell'Imperatore:

Quinci oltre io vo' che vedi a parte a parte quel ch'à natura accorta adoprar ivi (e mostrò la Cittade) hor è permesso da l'alto cielo dell'uno e l'altro sesso (Triompho, c. 33r).

Le lodi delle dame (la figlia dell'Imperatore in primis) si uniscono a quelle degli uomini, fidi consiglieri e condottieri di Carlo, in ritratti singoli o scene corali. Si veda ad esempio uno dei più bei ritratti femminili, quello della principessa di Bisignano:

Onde se veder brami un bel tesoro de la natura pria qui mira fiso.

Ved' il chiaro christal, vedi il fin'oro

l'hebano nero e il sol del Paradiso, i focosi rubini e il bianco avoro con le minute perle che nel viso raccolte stan qual vedi di costei formata per le man de li alti dei.

Qual bianca neve o qual vermiglia rosa venir a paregiarse mai potrebbbe con l'una e l'altra guancia gratiosa di lei, hor crederò che mai non crebbe

\footnotetext{
${ }^{18}$ Si veda a modello quest'ottava: "Ecco qui il vencitor, ecco quell'una alma fenice, fortunata tanto, a cui natura il cielo et la fortuna han dato largamente e donan quanto per lor donar si può ne mai alcuna cosa li pon mancar specchiati alquanto nel veramente imperial suo volto ov'ogni ben del mondo sia raccolto" (Triompho, c. 72v).
} 
ne i liti fortunati alcuna cosa

di più pregio o valor né so com'hebbe

tanto poco veder l'alma Natura

che di donarla al mondo hebb' ella cura (Triompho, c.35r).

La parte più interessante del testo è l'itinerario tra terrazze e palazzi, seguendo il percorso del trionfo attraverso i seggi (Capuano, Montagna, Nido, Popolo, Porta nuova e Porto) e descrivendo anche gli allestimenti che furono realizzati (ad esempio gli archi, le statue dei Colossi) per quella occasione.

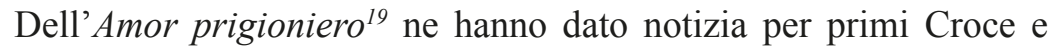
Ceci, poi grazie agli studi di Alfredo Parente (Parente, 1942), che ha pubblicato in edizione moderna il poemetto, disponiamo di più esaustive informazioni sull'opera e sull'autore. Mario Di Leo, pugliese, compose questo poemetto dopo la conquista di Tunisi ad opera dell'Imperatore, come testimoniato dai richiami nel testo ${ }^{20}$ poi lo fece pubblicare nel giugno del 1538 con dedica a Maria di Cardona.

Il modello è, come ipotizzato nelle Lodi di dame napoletane, il $\mathrm{Cu}$ pido cruci affixus dell'Ausonio. Si tratta di 229 ottave divise in due canti che presentano l'autore partecipe delle vicende che narra: Giove suscita l'ira di Venere quando decide di conferire alle donne partenopee "un sì bel trofeo lor tempie cinga / corona trionfal di sacro alloro", allora questa incita Cupido a vendicarsi di quelle "ninfe di Sebeto [...] che sprezzan del tuo fuoco il santo ardore, / sì vaghe e belle che fra lor

${ }^{19}$ L'Amor prigioniero, edito a Napoli nel 1538, risale secondo G. Ceci e B Croce al 1536. Ripubblicata in area veneziana nel 1550 (presso Valvassori) e nel 1551 (presso Bindoni), l'opera venne inserita nella Seconda parte delle Stanze, raccolta curata da Ludovico Dolce ed edita dal Giolito nel 1563, e nelle sue successive edizioni, riapparendo in tempi più recenti grazie al Croce. Cito dall'edizione curata da Parente, 1942: 151-217, 258-266.

${ }^{20}$ L'autore in riferimento a Margherita d'Austria la dice già duchessa di Firenze ("Arno io ti veggio di costei soggetto" Amor, II, ott. 66, v. 5) e poi per Carlo V:

"Io veggio Carlo che superbo esplica

le vele al vento e con le genti strane

turba la gente a me più ch'altra amica,

le libiche contrade e l'africane;

e vi rinova quella tema antica

ch'un tempo vi portâr l'arme romane;

ma chi contra costui può far difesa

non che vendetta di sì grave offesa" (Amor, II, ott. 31). 
non veggio / od occhio o volto onde non spiri amore" (Amor, I, ott. 23, vv. 1-4).

Cupido giunge così nei pressi di Miseno, costruisce il suo palazzo ed inizia a fare strage tra gli amanti tra cui, fatto prigioniero, anche l'autore. Incitate da Giunone che invia sulla terra Bellona e Diana, le ninfe si ribellano e catturano Amore che sarà liberato solo grazie all'intercessione di Giove invocato dalla madre.

Questa è la cornice che fa da sfondo al catalogo delle belle ninfe del secondo canto dove ritroviamo, a far da guida al poeta, un cavaliere probabilmente della famiglia dei Di Gennaro (è ipotesi in Ceci \& Croce, 1894: XVIII e nota) che, come la Beatrice di Beldando, gli dice "il nome di ciascuna / che scorgerò tra questa lieta gente, / senz'ordine serbar, perché amo ognuna / d'una fe" (Amor, II, ott. 53, vv. 1-4). Le dame sono le medesime che affollano gli altri poemetti coevi ma il Di Leo si distingue per l'utilizzo di un lessico meno formulare, le ottave sono più fluide tanto da ricordare a Croce quelle dell'Ariosto ${ }^{21}$, i riferimenti reali alla biografia delle donne e quelli fantastici al mito sono fusi in strutture serrate:

Quell'altra ch'io volea poc'anzi dirti, ch'era la bella figlia di Latona, conosco ai crini inanellati ed irti aver sembianza di Maria Cardona.

Oh che gioia n' avran tuoi vaghi spirti veggendo ora i begli occhi e la persona, se già la fama de le lode intese tutta di santo ardor l'alma t'accese!

Quest'è colei per la cui bionda testa riserbata vegg'io doppia corona: l'una per mano di Sebeto intesta, che per mercé di tal vittoria dona; l'altra che fanno con diletto e festa le sante abitatrici d'Elicona, perché farà sentir di Gange a Tile la dotta voce e l'onorato stile (Amor, II, ott. 70-71).

\footnotetext{
21 '“[...] l'eleganza della dizione, la musicalità del verso, l'agile costruzione dell'ottava. Il Di Leo, come parecchi altri poeti napoletani del tempo, si mostra in queste cose buon seguace dello stile del divino Ludovico" (Ceci \& Croce, 1894: XXI).
} 
La schiera delle guerriere si arricchisce anche delle dame di Barletta, conterranee dell'autore che in un rovesciamento di ruoli le presenta al cavaliere (II, ott. 121-122). Ad accomunare L'Amor prigioniero agli altri poemetti sono: le dichiarazioni di inadeguatezza dell'autore, che qui si arricchiscono con il riferimento ai poeti coevi (dal Molza al Martirano, II, ott. 3-11); alcune forme topiche della lode, come il paragone alle stelle o al sole (II, ott. 55, 73), o del ritratto femminile (hanno quasi tutte crini d'oro); il ricorso al valore metaforico dei nomi come "vittoria" o "colonna" (II, ott. 47,88 ) e alle formule di presentazione come "vedi" e simili.

Nella Clorida ${ }^{22}$ (1547) il Tansillo fa un breve cenno alle donne napoletane che formano lo stuolo al seguito dell'omonima ninfa (ott. 18-28):

E perché, senza belle, oneste e saggie donne, raro han diletto animi accorti; e fonti senza umor, senza fior piaggie son senza voi de gli uomini i diporti; squadra che a par del sol risplenda e raggie con voi ne venga, e meco si dirporti et con l'ostro de' volti et con l'avorio faccian vergogna a i fiori ond'io mi glorio (Clorida, ott. 18).

I riferimenti sono brevi e stereotipati: l'inserto catalogico è figlio della più vasta tradizione che qui stiamo analizzando, e in piccolo ne riprende le caratteristiche. Si veda ad esempio quest'ottava:

Due Spinelle che 'l mondo par ch'onori, vengano ad onorar le mie brigate;

spine che d'ogni tempo han frutti e fiori, fior di bellezze, e frutti d'onestate.

Vengavi la Monforte ch'à gli onori degli avi ha l'alte sue virtù agguagliate; e la sua figlia alteramente umana, ch'è nel nome et nel core vera Diana (Clorida, ott. 21).

\footnotetext{
${ }^{22}$ La Clorida in ms. Napoli, Biblioteca dei Gerolamini, cod. 210 (già XII. 9). Edita a stampa in [Stanze] Del signor Tansillo gentilhuomo di Sua Maestà. A l'illustr. et eccell. S. D. Pietro di Toledo, Viceré di Napoli, in Terminio, 1563. Cito da Flamini (1893: 115191). Il Tansillo, su imitazione dell'Aretusa, scrive un poemetto lirico-descrittivo che tratta dell'omonima ninfa che invita il padre di don Garcia, il viceré, a visitare la dimora del figlio. È un omaggio al suo signore e alla sua Napoli.
} 
Della seconda metà del Cinquecento è il poemetto Palagio d'Amore ${ }^{23}$ (1561) di Ludovico Paterno, che riprende motivi topici già sviluppati in altri testi come la predilezione degli dèi per la città partenopea e il motivo della costruzione del tempio-palazzo di Cupido. L'autore però, nella serie delle lodi ha il pregio di inserire parziali visioni della vita letteraria e cortigiana di Napoli attraverso un artificio nuovo per il genere. L'antefatto è il seguente: dopo aver chiesto a Febo quale sia il luogo più bello e più giocondo, Cupido si reca a Napoli e qui decide di costruire il suo palazzo, un edificio ornato d'oro, gemme, con colonne di diamante, archi di mosaico e palchi di marmo. C'è anche:

La ben capace e ricca sala, intorno di statue d'or perfettamente belle, piena era tutta, che vergogna e scorno facean' al torchio de l'ardenti stelle le quai sovr'altre, che più vago e adorno rendean quel luogo, havean nel lembo quelle lode intagliate, che si sparser fori per l'honorate penne de' migliori (Palagio, p. 302).

Ogni statua, in totale 16 , che rappresenta una nobildonna napoletana è quindi accompagnata da un'iscrizione che indica il poeta che l'ha cantata. Si veda ad esempio:

Gironima Colonna era la terza, figlia del grande Ascanio, e dopo questa quella seguia, che da mattino a terza, a nona, a vespro i chiari ingegni desta.

D’Aragona Isabella e seco scherza

Amor, né Gratia alcuna indietro resta

Minturno e Tasso eran de l'una carchi

e de l'altra il Tansillo e 'l dotto Varchi (Palagio, p. 303).

Amore potrà godersi la sua bella dimora finché "di Soliman le genti infide" ${ }^{24}$ vinceranno i sorrentini, allora Cupido abbandonerà Napoli alla volta della sua "prima culla".

\footnotetext{
${ }^{23}$ L'opera venne stampata ne Le nuove fiamme di Ludovico Paterno, Venezia, Valvassori, 1561 (II Libro). Cito da Terminio (1563: 297-306).

${ }^{24}$ Allusione a sacco dei Turchi a Sorrento del 1558.
} 
Scritti lontano da Napoli sono il Ritratto o modello delle grandezze, delitie et maraviglie della nobilissima città di Napoli (1588 ca.) ${ }^{25}$ di Giovan Battista del Tufo e i Capitoli delle bellezza (1605) $)^{26}$ di Filenio Pellegrino (pseudonimo di Marcello Macedonio). Il marchese Del Tufo decide "in omaggio alle cortesie ricevute dalle gentili Dame milanesi le quali gli fecero dimenticare tutte le amarezze subite in guerra tra $\mathrm{i}$ commilitoni e ed altrove tra amici e conoscenti" (Tagliareni, 1959: 12) di scrivere questo polimetro suddiviso in sette Ragionamenti. Vera protagonista è la città di Napoli, le sue tradizioni e i suoi abitanti tra cui le gentildonne descritte nel quarto Ragionamento.

In questo sono illustrati i più diffusi passatempi come i "diversi giuochi" di Carnevale, che offrono ai cavalieri l'opportunità di cimentarsi in varie prodezze e di essere ammirati dalle loro finestre dalle gentildonne reputate della città, qui racchiuse in un galante catalogo. Quello del Di Tufo è un ritratto mosso, le donne vengono esaltate per la loro bellezza dai balconi, mentre sono impegnate "scorgendo già venir, chi in mezzo o innante, / il fratello, il marito, o pur l'amante, / d'amor ciascuna accesa / conoscendo colui solo all'impresa" (Ritratto, IV, IX, vv. 15-18).

Le forme delle lode sono le canoniche, iperboliche (sembra che appaiano dai balconi nuovi soli, nuovi paradisi) ${ }^{27}$, e stilizzate, si veda ad esempio quello di Violante di Sangro e compagne:

Com'à l'uscir d'aprile, più sempre bella ne vago stelo, quando è più chiaro il cielo

tra gl'altri fior vermigli:

rose, ligustri e gigli

vaga al color la semplice viola, tal questa Violante al mondo sola.

\footnotetext{
${ }^{25}$ Il Ritratto è tràdito dal ms. XIII. C. 96. (Biblioteca Nazionale di Napoli); l'opera di Del Tufo è stata anche edita da C. Tagliareni, 1959. Cito da questa edizione. Ho numerato i componimenti del "ragionamento" da I (Donne illustri che compariscono in Carnevale alle finestre) a XVII (Barriere e barreggianti vestiti di colori diversi per Carnevale).

${ }^{26}$ L'opera venne stampata con titolo Capitoli della bellezza a Venezia con editore G. B. Ciotti nel 1605. In edizione moderna sulla base dell'edizione del 1615 in Borzelli,1895. Cito da Borzelli, 1895.

${ }^{27}$ Cfr. Ritratto di Margherita d'Ávalos d'Aragona, Giovanna Pignatelli, Beatrice di Giovara, Camilla d'Afflitto, Isabella Gesualdo.
} 
Dal'altra parte poi di quella strada

dove mirar più a grata

vedrà se veder brama

altre tante celesti e gloriose

come tra gigli le vermiglie rose

su i color variati

di drappi e di broccati

da far gl'amori degl'odi innamorati (Ritratto, IV, V, vv. 21-35).

Marcello Macedonio, innamoratosi di una nobildonna napoletana già sposata, tale Isabella Sanseverino ${ }^{28}$, è costretto ad allontanarsi da Napoli e a Venezia pubblica i suoi Capitoli in lode della bellezza della donna amata e delle altre donne, napoletane e non. Il poemetto, in terzine dantesche, contiene l'elogio di dame contemporanee, "secondo lo schema barocco della galleria" (De Miranda):

Dall'elenco, che risulta partenopeo e romano, veneziano e più globalmente, italiano, europeo è il ritratto di una nobiltà che senza dubbio costruisce il suo vanto con le imprese degli eroi, le dimore e le collezioni d'arte ma anche edifica altari per sé stessa con le figure splendenti delle sue dame di casa, voci consolatorie e consigliere sagge, occhi che vigilano, corpi sinuosi che procreano e, in particolar modo nei Capitoli, alabastri avvolti in vesti preziose, automi miracolosi, volti che infiammano l'ispirazione di letterati... (De Miranda, 2008: 265-66).

Il secondo capitolo intitolato Della bellezza napolitana si apre con la descrizione della città ricordata dall'esule nella foggia della sua fondatrice Partenope ("Quasi donna regal su ricco seggio", II, v. 9), segue "Io de le belle sue prendo a cantare" per poi concludere con la sua donna "Isabella s'e fior de le altre belle" (II, v. 250). Si leggano ad esempio le terzine dedicate a Lucrezia Cattinaro:

Segue tal, cui mirando io mi confondo,

Amor dentro i begli occhi nacque e crebbe,

Beltà con lei d'un parto uscì nel mondo.

Chi del viso leggiadro a pien direbbe?

Più felice Lucrezia, a lei s'inchini

L'altra, a cui l'onor per la vita increbbe

${ }^{28}$ Secondo l'ipotesi di Borzelli (1895: 12-20). 
Trasse famoso Eroe fin da' confini,

Che chiude il Pireneo, l'Ibero bagna,

Forza ch'uscio da gli occhi suoi divini (Capitoli, II, vv. 31-39).

\subsection{Il rovescio della lode: $i$ cataloghi satirici}

Le opere a stampa intorno alla nobiltà napoletana per aver troppo falsato la realtà e dipinto quella raffinata corte come modello di perfezione, fisica e morale, suscitarono la reazione di autori che, decisi a raccontare la Verit $^{29}$, fecero circolare le loro opere manoscritte:

$[\ldots]$ così quel genere di produzione $[\ldots]$ trovò altri spiriti bizzarri che vollero di proposito presentar l'altro lato della bella medaglia; e come, quelli togliendo come pretesto dalla bellezza dissero delle virtù di ciascuna, così questi, movendo d'altro fine, rilevarono e rivelarono solo la parte brutta e, per reazione, nelle colpe di quelle infierirono con poca galanteria (Borzelli, 1908: 235).

Menzionata da Croce a titolo d'esempio è l'opera Le Metamorfosi del Secolo XVIII $I^{30}$ in cui sotto travestimenti mitologici non troviamo più donne caste e devote ma libertine ad esempio in riferimento a Donna Carolina Sparnocchi:

Qui non fedel Penelope

Fredda di amore al foco

De Proci suoi l'insidie

Fugge e mantiene in gioco.

Scorra ramingo il fluido

Regno l'accorto sposo

Il lungo dì non passasi

\footnotetext{
${ }^{29}$ Cfr. i mss. dei fratelli Silvio e Ascanio Corona: La Verità svelata a' Principi ovvero Successi diversi tragici ed amorosi occorsi in Napoli o altrove a Napolitani, cominciando dalli Re Aragonesi. Composta dalli dottori Silvio ed Ascanio Corona (Biblioteca Nazionale di Napoli X. C. 19). Casi tragici et amorosi successi a famiglie napolitane incominciando dall'anno 1442 sin al 1680 (Biblioteca Nazionale di Napoli X. C. 15). Utile strumento alla consultazione dei numerosi manoscritti (ne ho riportati solo due ad esempio) che testimoniano l'opera dei Corona sono Borzelli, 1891; Borzelli, 1908. ${ }^{30} \mathrm{Cfr}$. Le metamorfosi del Secolo XVIII che trovansi dipinte da varij celebri autori nella Galleria del sig. D. Salvatore Contorto e Donna Francesca Maria Catenaccio. Anacreontica (bib Naz. Ms. XV. D. 1.). Cito da Borzelli (1908: 241-259).
} 
Per lei freddo e noioso.

Allor che il drappo credesi

Che tesse e che discioglie

Un amator sollecito nelle sue braccia accoglie (Metamorfosi, vv. 340$348)$.

Nel Seicento, scemata la galanteria e l'adulazione a causa di un mutamento nei sentimenti sociali (come afferma Croce cambia il modo di amare $^{31}$ ), le donne scendono dai loro troni reali e celestiali dove "l'egoismo maschile le aveva collocate" sancendo di fatto la fine dei cataloghi elogiativi nella forma che aveva avuto tanto successo nella Napoli cinquecentesca.

\section{Bibliografia e abbreviazioni}

Altamura, Antonio (ed.). 1972. Lo specchio delle bellissime donne napoletane di Iacomo Beldando. In Curiosità letterarie napoletane. Terza serie. Napoli: Edizioni scientifiche napoletane, 17-62.

Altamura, Antonio (ed.). 1980. Il "Tempio d'Amore". Storia di un plagio. Napoli: Società editrice napoletana. [Tempio].

Ascarelli, Fernanda. 1953. La tipografia cinquecentina italiana. Firenze: Sansoni Antiquariato.

Ascarelli, Fernanda \& Menato, Marco. 1989. La tipografia del '500 in Italia. Firenze: Leo S. Olschki.

Borzelli, Angelo (ed.). 1895. I capitoli della bellezza di Marcello Macedonio (Filenio Pellegrino). Napoli: G. Stanziola. [Capitoli].

Borzelli, Angelo. 1888. Una poetessa del secolo XVI (Gaspara Stampa (15231553)). Napoli: Chiurazzi.

Borzelli, Angelo. 1891. Notizia dei mss. Corona ed il successo di "d. Maria d'Avolos principessa di Venosa e di Fabrizio Carafa duca d'Andria" illustrato dalle poesie dei contemporanei. Torino: G. B. Paravia.

Borzelli, Angelo. 1908. Le metamorfosi del Secolo XVIII che trovansi dipinte da varij celebri autori nella Galleria del sig. D. Salvatore Contorto e Donna Francesca Maria Catenaccio. Anacreontica (bib Naz. Ms. XV. D. 1.). In Borzelli, Angelo (ed.) Successi tragici et amorosi di Silvio et Ascanio Corona. Napoli: F. Casella, 241-259. [Metamorfosi].

Branca, Vittore. 1967. Introduzione. In Branca, Vittore (ed.) Caccia di Diana, in Tutte le opere di Giovanni Boccaccio I. Milano: Mondadori, 3-13.

${ }^{31}$ Cfr. Ceci \& Croce, 1894: XI. 
Bresciano, Giovanni. 1936. Neapolitana II. Nuovi contributi alla storia della tipografia napoletana nel secolo XVI. Firenze: Leo S. Olschki.

Bruni, Francesco. 2002. L'italiano letterario nella storia. Bologna: Il Mulino.

Ceci, Giuseppe \& Croce, Benedetto. 1894. Lodi di dame napoletane del secolo decimosesto dall' "Amor prigioniero" di Mario Di Leo, con notizie ed estratti di altri poemetti sincroni di simile argomento. Napoli.

Croce, Benedetto. [1942] 1953. Aneddoti di varia letteratura I. Bari: Laterza.

D’Agostino, Guido. 1967. Il Governo Spagnolo nell' Italia meridionale (Napoli dal 1503 al 1580). In AA. VV. Storia di Napoli V. Napoli: Società editrice Storia di Napoli, tomo I, 3-160.

De Blasi, Nicola \& Varvaro, Alberto. 1988. Napoli e l'Italia meridionale. In Asor Rosa, Alberto (dir.) Letteratura Italiana. Storia e Geografia II, L'età moderna. Torino: Einaudi, tomo I, 235-325.

De Miranda, Girolamo. 2008. Marcello Macedonio, Capitoli della bellezza (1605). In Caruso, Carlo \& Spaggiari, William (coord.) Filologia e storia letteraria. Studi per Roberto Tissoni. Roma: Edizioni di storia e letteratura, 263-271.

De Nichilo, Mauro. 2006. Poesia volgare a Napoli nel 1536: Lo "Specchio de le bellissime donne napoletane" di Giacomo Beldando. In Guaragnella, Pasquale \& Santagata, Marco (coord.) Studi di letteratura italiana. Per Vitilio Masiello. Roma-Bari: Laterza, 351-380.

Dilemmi, Giorgio. 2000. Da Mantova a Napoli: i "pretesti” asolani di Giovanni Muzzarelli e Augostino Landulfo. In Dilemmi, Giorgio. Dalle corti al Bembo. Bologna: CLUEB, 287-301.

Dionisotti, Carlo. 1963. Appunti sulle rime del Sannazaro. Giornale storico della letteratura italiana CLX: 78-131.

Epicuro, Marcantonio (ed.). 1942. L'Amore prigioniero. In Parente, Alfredo (a cura di). I drammi e le poesie italiane e latine aggiuntovi l'Amore prigioniero di Mario Di Leo. Bari: Laterza, 151-217, 258-266. [Amor].

Fera, Vincenzo. 2001. Tra piemontesi ad Oxford. La lectureship di Dionisotti. In Fumagalli, Edoardo (a cura di). Carlo Dionisotti, geografia e storia di uno studioso. Roma: Edizioni di Storia e Letteratura, 25-46.

Ferrero, Ermanno \& Müller, Giuseppe. 1892. Carteggio di Vittoria Colonna marchesa di Pescara, seconda edizione con supplemento raccolto e annotato da D. Tordi. Torino: Loescher.

Flamini, Francesco. 1893. La Clorida. In Flamini, Francesco (ed.) L'egloga e $i$ poemetti di Luigi Tansillo, con introduzione e note di F. Flamini. Napoli [ma Trani: pei tipi del Cav. V. Vecchi], 115-191. [Clorida].

Galasso, Giuseppe. 2006. L'età di Carlo V. In Galasso, Giuseppe. Storia del Regno di Napoli II. Torino: Utet, 309-550. 
Giuliani, Luigi. (ed.). 2004. Poemas de Juan de Tapia. Salamanca: Ediciones Universidad de Salamanca. [Poemas].

Giustiniani, Lorenzo. 1793. Saggio storico-critico sulla tipografia del Regno di Napoli. Napoli: Orsini.

Manzi, Pietro. 1970. Annali di Giovanni Sultzbach: (Napoli, 1529-154 Capua, 1547). Firenze: Leo S. Olschki.

Manzi, Pietro. 1972. La tipografia napoletana nel '500: annali di Mattia Cancer ed eredi:(1529-1595). Firenze: L. S. Olschki.

Mazzuchelli, Giammaria M. 1753. Gli scrittori d'Italia, cioè notizie storiche e critiche intorno alla vita e gli scritti dei letterati italiani I. Brescia: presso G. B. Bossini.

Parenti, Giovanni. 1995. Un cultore cinquecentesco di Dante e Petrarca: Ioan Berardino Fuscano. In Magnani, Franca (cur.) Studi in memoria di Paola Medioli Masotti. Napoli: Loffredo.

Periñán, Blanca (ed.). 1968. Las poesías de Suero de Ribera. Estudio y edición critíca anotada de los textos. Miscellanea di studi ispanici XVI. Pisa: Istituto di Lingua e Letteratura Spagnola dell’Università di Pisa: 5-138. [Poesías].

Petrocchi, Giorgio. 1967. La letteratura del pieno e del tardo Rinascimento. In AA. VV. Storia di Napoli V. Napoli: Società editrice Storia di Napoli, tomo I, 281-322.

Pino, Giovanni Battista. 1536. Il Triompho di Carlo Quinto a cavallieri et alle donne napoletane. Napoli: I. Sultzbach. [Triompho].

Raccolta di Poesie diverse, ms. XIII. G. 42., Biblioteca Nazionale di Napoli, miscellaneo, cc. 231r-241v.

Ruotolo, Roberta Angelica (ed.). Lo specchio de le bellissime donne napoletane di Iacomo Beldando: Edizione critica (tesi di Laurea Magistrale). Napoli: Università degli studi di Napoli-Dipartimento di studi umanistici (in pubblicazione). [Specchio].

Santoro, Marco. 1997. Editoria e cultura a Napoli nel Cinquecento. Accademie e biblioteche italiane LXV(1): 5-24.

Tagliareni, Calogero (ed.). 1959. Ritratto o modello delle grandezze, delitie e meraviglie della nobilissima città di Napoli. Testo inedito del Cinquecento di Giovan Battista del Tufo. Napoli: Agar. [Ritratto].

Tagliareni, Calogero. 1954. Opera manoscritta del Marchese G.B. Del Tufo poeta napoletano del Cinquecento (Usi e costumi, spassi, giuochi e feste in Napoli). Napoli: Pironti.

Tagliareni, Calogero. 1959. Introduzione. In Tagliareni, Calogero (ed.) Ritratto o modello delle grandezze, delitie e meraviglie della nobilissima città di Napoli. Testo inedito del Cinquecento. Napoli: Agar, 7-18. 
Terminio, Antonio. 1563. Il Palagio d'Amore. In Terminio, Antonio (ed.) La seconda parte delle Stanze di diversi auttori nuouamente mandata in luce alla nobiliss. Signora Camilla Imperiale. Venezia: Giolito, 297306. [Palagio].

Toscano, Tobia R. 1992. Contributo alla storia della tipografia a Napoli nella prima metà del Cinquecento (1503-1553). Napoli: Ente regionale per il diritto allo studio universitario "Napoli 1".

Toscano, Tobia R. 2000. Carlo V nelle delizie aragonesi di Poggio Reale. In Toscano, Tobia R. Letterati corti accademie. La letteratura a Napoli nella prima metà del Cinquecento. Napoli: Loffredo, 245-263.

Zappella, Giuseppina. 1984. Tipografia campana del Cinquecento: centri e stampatori. Dizionario storico- bibliografico. Napoli: Accademia Pontaniana. 